\title{
Adaptive Fuzzy Output Feedback Control based on Observer for Nonlinear Heating, Ventilating and Air Conditioning System
}

\author{
Jaeho Baek, Eun-ju Hwang, Euntai Kim and Mignon Park* \\ Department of Electrical and Electronics Engineering, Yonsei University, Seoul, Korea
}

\begin{abstract}
A Heating, Ventilating and Air Conditioning (HVAC) system is a nonlinear multi-input multi-output (MIMO) system. This system is very difficult to control the temperature and the humidity ratio of a thermal space because of complex nonlinear characteristics. This paper proposes an adaptive fuzzy output feedback control based on observer for the nonlinear HVAC system. The nonlinear HVAC system is linearized through dynamic extension. State observers are designed for estimating state variables of the HVAC system. Fuzzy systems are employed to approximate uncertain nonlinear functions of the HVAC system with unavailable state variables. The obtained controller compares with an adaptive feedback controller. Simulation is given to demonstrate the effectiveness of our proposed adaptive fuzzy method.
\end{abstract}

Key Words : Adaptive Control, Fuzzy Control, MIMO System, temperature control, humidity control, HVAC System.

\section{Introduction}

A HVAC system includes all Air-Conditioning system used for cooling and heating the room or buildings. The energy consumed by HVAC systems in commercial and industrial buildings constitutes more $50 \%$ of the world energy consumption [1]. A mere $1 \%$ improvement in energy efficiency of these systems translates into annual savings of millions of dollars at the national level [2]. The literatures in the design of controller for a HVAC system shows different researches to fully or partially achieve controlling the temperature and humidity ratio.

In [1], an observer was proposed in estimating the thermal load and moisture load. In [6], feedback linearization was applied to a HVAC model and the actuator' $\mathrm{s}$ dynamics was considered. Several intelligent methods were also used for control of a HVAC system in [7-8]. In [9], an adaptive and robust controller was designed for a nonlinear HVAC system with unknown parameters. In spite of the development of new control methodologies for a HVAC system aiming at improving their energy efficiencies, the process of operating a HVAC system is still low efficiency and high energy consumption process [3]. For many years, PID and direct digital controls have also been used for temperature and humidity ratio control [4][5]. The developed HVAC control techniques are insufficient for the complex nonlinear characteristics of a MIMO HVAC system with unavailable state variables.

In this paper, we propose an adaptive fuzzy output feedback

Manuscript received Sep. 30. 2008; revised Apr. 9. 2009.

* Corresponding Author : mignpark@yonsei.ac.kr

This research was supported by a grant (06Construction CoreB02) from High-tech Urban Development Program (HUDP) funded by Ministry of Land, Transport and Maritime Affairs of Korea Government. controller based on observer for the HVAC system in achieving a good heating, ventilating and air conditioning performance. The dynamic extension concept of [7] is used in feedback linearization mothod. Fuzzy systems is also used to approximate the uncertain functions of the HVAC system with unavailable state variables. The state observer is constructed for estimating unavailable state variables. The proposed adaptive fuzzy control method maintains the desired temperature and humidity ratio in the HVAC system.

The remainder of this paper will be organized as follows. Section 2 will introduce a HVAC system, a dynamic equation and a state space model with actuator dynamics. In section 3, we will present the feedback linearization technique with dynamic extension algorithm for a nonlinear HVAC system. An adaptive fuzzy output feedback control based on observer will be introduced for the unavailable state variables of a HVAC system in section 4. Fuzzy systems will be briefly presented in this section. In section 5, simulation results of the adaptive fuzzy output feedback control method will be given. Finally, section 6 will give some concluding remarks.

\section{HVAC System}

We consider the single-zone HVAC system shown as Fig. 1.

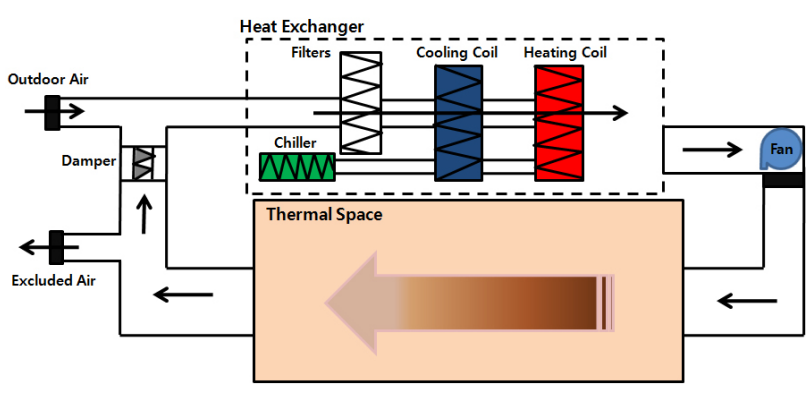

Fig. 1 HVAC System 
The dynamic equation of the HVAC system is derived from energy conservation principle [1] and are given by

$$
\begin{aligned}
& \dot{T}_{3}=\frac{60 f_{r}}{V_{s}}\left(T_{2}-T_{3}\right)-\frac{60 h_{f g} f_{r}}{c_{p} V_{s}}\left(W_{s}-W_{3}\right)+\frac{\left(Q_{o}-h_{f g} M_{o}\right)}{(1-\mu) \rho_{a} c_{p} V_{s}} \\
& \dot{W}_{3}=\frac{60 f_{r}}{V_{s}}\left(W_{s}-W_{3}\right)+\frac{M_{o}}{\rho_{a} V_{s}} \\
& \dot{T}_{2}=\frac{60 f_{r}}{V_{h e}}\left(T_{3}-T_{2}\right)-\frac{60(1-\mu) f_{r}}{V_{h e}}\left(T_{o}-T_{3}\right) \\
& -\frac{60 h_{w} f_{r}}{c_{p} V_{h e}}\left\{(1-\mu) W_{o}+\mu W_{3}-W_{s}\right\}-6000 \frac{g p m}{\rho_{a} c_{p} V_{h e}}
\end{aligned}
$$

The state variable form of the dynamic equation (1) can be rewritten as

$$
\begin{aligned}
& \dot{z}_{1}=u_{1} \alpha_{1}\left(z_{3}-z_{1}\right)-u_{1} \alpha_{2}\left(W_{s}-z_{2}\right)+\alpha_{3}\left(Q_{o}-h_{f g} M_{o}\right) \\
& \dot{z}_{2}=u_{1} \alpha_{1}\left(W_{s}-z_{2}\right)+\alpha_{4} M_{o} \\
& \dot{z}_{3}=u_{1} \beta_{1}\left(z_{1}-z_{3}\right)+(1-\mu) u_{1} \beta_{1}\left(T_{o}-z_{1}\right) \\
& -u_{1} \beta_{3}\left\{(1-\mu) W_{o}+\mu x_{2}-W_{s}\right\}-6000 u_{2} \beta_{2}
\end{aligned}
$$

where

$u_{1}=f_{r}, u_{2}=g p m, z_{1}=T_{3}, z_{2}=W_{3}, z_{3}=T_{2}, \quad \alpha_{1}=\frac{60}{V_{s}}$, $\alpha_{2}=\frac{60 h_{f g}}{c_{p} V_{s}}, \quad \alpha_{3}=\frac{1}{(1-\mu) \rho_{a} c_{p} V_{s}}, \quad \alpha_{4}=\frac{60}{\rho_{a} V_{s}}, \quad \beta_{1}=\frac{60}{V_{h e}}$, $\beta_{2}=\frac{1}{\rho_{a} c_{p} V_{h e}}$ and $\beta_{3}=\frac{60 h_{w}}{c_{p} V_{h e}}$.

In addition, the control input signals $\mathbf{u}=\left[\begin{array}{ll}u_{1} & u_{2}\end{array}\right]^{T}$ in the system (2) are implemented to liquid valves [10]. The valve dynamic model can consider as

$$
u_{1}=\frac{k_{1}}{1+\tau_{1} S} v_{1}, \quad u_{2}=\frac{k_{2}}{1+\tau_{2} S} v_{2}
$$

where $k_{1}, k_{2}, \tau_{1}$ and $\tau_{2}$ are the actuator's gain and time constant. $\mathbf{u}=\left[\begin{array}{ll}u_{1} & u_{2}\end{array}\right]^{T}$ is the control signal applied to the HVAC system and $\mathbf{v}=\left[\begin{array}{ll}v_{1} & v_{2}\end{array}\right]^{T}$ is the input signal applied to the actuator. Therefore, we derive an augmented state space model with the new state vector as

$$
\mathbf{z}=\left[\begin{array}{lllll}
z_{1} & z_{2} & z_{3} & u_{1} & u_{2}
\end{array}\right]^{T}=\left[\begin{array}{lllll}
z_{1} & z_{2} & z_{3} & z_{4} & z_{5}
\end{array}\right]^{T} .
$$

The system model (2) becomes

$$
\begin{aligned}
& \dot{\mathbf{z}}=\mathbf{f}(\mathbf{z})+\mathbf{g}(\mathbf{z}) \mathbf{v}=\left[\begin{array}{c}
a_{1}(\mathbf{z}) \\
a_{2}(\mathbf{z}) \\
a_{3}(\mathbf{z}) \\
a_{4}(\mathbf{z}) \\
a_{5}(\mathbf{z})
\end{array}\right]+\left[\begin{array}{cc}
0 & 0 \\
0 & 0 \\
0 & 0 \\
\frac{k_{1}}{\tau_{1}} & 0 \\
0 & \frac{k_{2}}{\tau_{2}}
\end{array}\right] \mathbf{v} \\
& \mathbf{y}=\left[\begin{array}{ll}
z_{1} & z_{2}
\end{array}\right]^{T}
\end{aligned}
$$

where

$a_{1}(\mathbf{z})=\left[\alpha_{1}\left(z_{3}-z_{1}\right)-\alpha_{2}\left(W_{s}-z_{2}\right)\right] u_{1}+\alpha_{3}\left(Q_{o}-h_{f g} M_{o}\right)=\gamma_{1} u_{1}$,
$a_{2}(\mathbf{z})=\alpha_{1}\left(W_{s}-z_{2}\right) u_{1}+\alpha_{4} M_{o}=\gamma_{2} u_{1}+\alpha_{4} M_{o}$,

$a_{3}(\mathbf{z})=\left[\beta_{1}\left(z_{1}-z_{3}\right)+(1-\mu) \beta_{1}\left(T_{o}-z_{1}\right)\right] u_{1}$

$+\left[-\beta_{3}\left\{(1-\mu) W_{o}+u z_{2}-W_{s}\right\}\right] u_{1}-6000 \beta_{2} u_{2}=\gamma_{3} u_{1}+\gamma_{4} u_{2}$,

$a_{4}(\mathbf{z})=-\frac{u_{1}}{\tau_{1}}$ and $\quad a_{5}(\mathbf{z})=-\frac{u_{2}}{\tau_{2}}$.

\section{Feedback Linearization for HVAC System}

We apply a feedback linearization technique to the augmented state space model (4) to track the desired temperature and humidity ratio. The HVAC system (4) has the relative degrees $\mathbf{r}=\{2,2\}$, which is the smallest number of times that the outputs $z_{1}$ and $z_{2}$ have to differentiate such that at least one of the inputs appears in $z_{1}{ }^{(2)}$ and $z_{2}{ }^{(2)}$ [12].

However, the decoupling matrix $\left[\begin{array}{cc}L_{g_{1}} L_{\mathbf{f}} z_{1} & L_{g_{2}} L_{\mathbf{f}} z_{1} \\ L_{g_{1}} L_{\mathbf{f}} z_{2} & L_{g_{2}} L_{\mathbf{f}} z_{2}\end{array}\right]$ is singular. The system (4) has no relative degree. To achieve the relative degree and non-interacting control, we employ feedback linearization technique with a dynamic extension [7].

We set $v_{1}=\varphi_{1}, \dot{\varphi}_{1}=\varepsilon_{1}$ and $v_{2}=\varphi_{2}$. The new augmented state variables are defined as $\overline{\mathbf{z}}=\left[\mathbf{z}, v_{1}\right]^{T} \in \mathbf{R}^{6}$ and the HVAC system will be composed as

$$
\dot{\overline{\mathbf{z}}}=\overline{\mathbf{f}}(\overline{\mathbf{z}})+\bar{g}_{1}(\overline{\mathbf{z}}) \varphi_{1}+\bar{g}_{2}(\overline{\mathbf{z}}) \varphi_{2}=\left[\begin{array}{c}
a_{1} \\
a_{2} \\
a_{3} \\
a_{4}+\frac{k_{1} z_{2}}{\tau_{2}} \\
a_{5} \\
0
\end{array}\right]+\left[\begin{array}{cc}
0 & 0 \\
0 & 0 \\
0 & 0 \\
0 & 0 \\
0 & \frac{k_{2}}{\tau_{2}} \\
1 & 0
\end{array}\right]\left[\begin{array}{l}
\varphi_{1} \\
\varphi_{2}
\end{array}\right]
$$

Following the procedure of linearization, system (5) can be rewritten as

$$
\begin{aligned}
z_{1}^{(3)} & =f_{1}(\mathbf{z})+g_{11}(\mathbf{z}) v_{1}+g_{12}(\mathbf{z}) v_{2} \\
& =L_{\overline{\mathbf{f}}}{ }^{3} z_{1}+L_{\bar{g}_{1}} L_{\overline{\mathbf{f}}}{ }^{2} z_{1} v_{1}+L_{\bar{g}_{2}} L_{\overline{\mathbf{f}}}{ }^{2} z_{1} v_{2} \\
z_{2}{ }^{(3)} & =f_{2}(\mathbf{z})+g_{21}(\mathbf{z}) v_{1}+g_{22}(\mathbf{z}) v_{2} \\
& =L_{\overline{\mathbf{f}}}{ }^{3} z_{2}+L_{\bar{g}_{1}} L_{\overline{\mathbf{f}}}{ }^{2} z_{2} v_{1}+L_{\bar{g}_{2}} L_{\overline{\mathbf{f}}}{ }^{2} z_{2} v_{2}
\end{aligned}
$$

where $\quad L_{\bar{g}_{1}} L_{\overline{\mathbf{f}}}^{2} z_{1}=\frac{\gamma_{1} k_{1}}{\tau_{1}} \quad, \quad L_{\bar{g}_{2}} L_{\overline{\mathbf{f}}}^{2} z_{1}=\frac{\alpha_{1} \gamma_{4} x_{4} k_{2}}{\tau_{2}}$, $L_{\bar{g}_{1}} L_{\overline{\mathbf{f}}}^{2} z_{2}=\frac{\gamma_{2} k_{1}}{\tau_{1}}$ and $L_{\bar{g}_{2}} L_{\overline{\mathbf{f}}}^{2} z_{2}=0$.

Equation (6) is equivalent to the following system

$$
\begin{aligned}
\dot{\boldsymbol{x}} & =\boldsymbol{A} \boldsymbol{x}+\boldsymbol{B}[\boldsymbol{F}(\boldsymbol{x})+\boldsymbol{G}(\boldsymbol{x}) \varphi] \\
\boldsymbol{y} & =\left[\begin{array}{ll}
x_{1} & x_{2}
\end{array}\right]^{T}
\end{aligned}
$$

where $x=\left[\begin{array}{llllll}x_{1} & x_{1}{ }^{(1)} & x_{1}{ }^{(2)} & x_{2} & x_{2}{ }^{(1)} & x_{2}{ }^{(2)}\end{array}\right]^{T}$, 


$$
\begin{aligned}
& \mathbf{F}(\mathbf{x})=\left[\begin{array}{ll}
f_{1} & f_{2}
\end{array}\right]^{T}, \mathbf{G}(\mathbf{x})=\left[\begin{array}{ll}
g_{11} & g_{12} \\
g_{21} & g_{22}
\end{array}\right], \\
& \mathbf{A}=\operatorname{diag}\left(\left[\begin{array}{lll}
0 & 1 & 0 \\
0 & 0 & 1 \\
0 & 0 & 0
\end{array}\right],\left[\begin{array}{lll}
0 & 1 & 0 \\
0 & 0 & 1 \\
0 & 0 & 0
\end{array}\right]\right), \mathbf{B}=\operatorname{diag}\left(\left[\begin{array}{l}
0 \\
0 \\
1
\end{array}\right],\left[\begin{array}{l}
0 \\
0 \\
1
\end{array}\right]\right) \text { and } \\
& \mathbf{C}=\operatorname{diag}\left(\left[\begin{array}{lll}
1 & 0 & 0
\end{array}\right],\left[\begin{array}{lll}
1 & 0 & 0
\end{array}\right]\right) .
\end{aligned}
$$

For the given set point references $y_{1 m}$ and $y_{2 m}$, we define the tracking errors as $e_{1}=y_{1}-y_{1 m}$ and $e_{2}=y_{2}-y_{2 m} \quad$ and denote as $\quad \mathbf{E}=\left[\begin{array}{ll}e_{1} & e_{2}\end{array}\right]^{T}$, $\mathbf{y}_{\mathbf{m}}=\left[\begin{array}{ll}y_{1 m} & y_{2 m}\end{array}\right]^{T}, \quad \mathbf{y}_{\mathbf{m}}{ }^{(3)}=\left[y_{1 m}{ }^{(3)} y_{2 m}{ }^{(3)}\right]^{T} \quad$ and $\mathbf{Y}_{\mathbf{m}}=\left[\begin{array}{llllll}y_{1 m} & y_{1 m}{ }^{(1)} & y_{1 m}{ }^{(2)} & y_{2 m} & y_{2 m}{ }^{(1)} & y_{2 m}{ }^{(2)}\end{array}\right]^{T} . \quad \mathrm{We}$ obtain the error matrix $\mathbf{e}=\mathbf{Y}_{\mathbf{m}}-\mathbf{x}=\left[\begin{array}{llllll}e_{1} & e_{1}{ }^{(1)} & e_{1}{ }^{(2)} & e_{2} & e_{2}{ }^{(1)} & e_{2}{ }^{(2)}\end{array}\right]^{T}$.

When the feedback linearization control law for HVAC system is designed as

$$
\mathbf{v}=\mathbf{G}^{-1}\left(-\mathbf{F}+\mathbf{y}_{\mathbf{m}}{ }^{(3)}-\mathbf{K e}\right)
$$

with the $\mathbf{K}=\operatorname{diag}\left(\left[\begin{array}{lll}k_{11} & k_{12} & k_{13}\end{array}\right],\left[\begin{array}{lll}k_{21} & k_{22} & k_{23}\end{array}\right]\right)$ chosen so that the polynomial $s^{3}+k_{i 1} s^{2}+k_{i 2} s+k_{i 3}=0, \mathrm{i}=1,2$ has all their roots strictly in the left-half complex plane, leads to meet the desired performance specification such as transient response or steady state error.

\section{Adaptive Fuzzy Output Feedback Control based on Observer for HVAC System}

The control law (8) is based on exact cancellation of the nonlinear terms. If HVAC system has $\mathbf{F}$ and $\mathbf{G}$ under the unavailable state variables, cancellation is not accuracy and the equation (7) is not linear.

Assumption. The dynamics (1) of HVAC system is too complex and uncertain for a mathematical model to describe. The state variables is not available for measurement.

In this section, we present an adaptive fuzzy output feedback controller to solve the problems. A sound solution would be to eliminate the uncertain term by using the approximation property of the fuzzy logic systems and designing an observer [11]. In the following, we briefly explain the approximation property of the fuzzy logic systems.

The basic configuration of fuzzy logic systems consists of some fuzzy IF-THEN rules and a fuzzy inference engine. The fuzzy inference engines are used to combine the fuzzy IFTHEN rules in the fuzzy rule base into a mapping from an input linguistic vector to an output linguistic variable. The ith fuzzy IF-THEN rule can be written as

$$
R^{l} \text { : if } x_{1} \text { is } A_{1}^{l}, x_{2} \text { is } A_{2}^{l}, \ldots \text {, and } x_{n} \text { is } A_{n}{ }^{l}
$$
then $y$ is $B^{l}$. where $A_{1}^{l}, A_{2}^{l}, \ldots, A_{n}{ }^{l}$ and $B^{l}$ are fuzzy sets.

The general fuzzy logic systems with singleton fuzzifier, product inference and center-average defuzzifier is designed as

$$
f(\mathbf{x})=\frac{\sum_{l=1}^{M} y^{l}\left[\prod_{i=1}^{N} u_{A_{i}^{l}}\left(x_{i}\right)\right]}{\sum_{l=1}^{M}\left[\prod_{i=1}^{N} u_{A_{i}^{l}}\left(x_{i}\right)\right]}
$$

where $\mathrm{M}$ is the number of the fuzzy rules, and $y^{i}$ is the point at which $u_{B^{l}}\left(\bar{y}^{l}\right)=1$.

MIMO fuzzy logic systems based on observer are of the form

$$
\begin{aligned}
& \hat{\boldsymbol{F}}\left(\hat{\boldsymbol{x}} \mid \boldsymbol{\Theta}_{1}\right)=\boldsymbol{\Phi}(\hat{\boldsymbol{x}}) \boldsymbol{\Theta}_{1} \\
& \hat{\boldsymbol{G}}\left(\hat{\boldsymbol{x}} \mid \boldsymbol{\theta}_{2}\right)=\boldsymbol{\Phi}(\hat{\boldsymbol{x}}) \boldsymbol{\theta}_{2}
\end{aligned}
$$

where $\boldsymbol{\Theta}_{1}$ and $\boldsymbol{\theta}_{2}$ are parameter vectors and $\boldsymbol{\Phi}(\hat{\mathbf{x}})=\operatorname{diag}\left(\xi^{T}, \xi^{T}\right)$ is a regressive vector.

Since we assume that the state variables for the HVAC system are unavailable for measurement, the state $\mathbf{x}$ and the error $\mathbf{e}$ replace their estimates $\hat{\mathbf{x}}$ and $\hat{\mathbf{e}}=\mathbf{Y}_{\mathbf{m}}-\hat{\mathbf{x}}$. Design the fuzzy adaptive observer for estimates $\hat{\mathbf{x}}$ as follows :

$$
\begin{aligned}
& \dot{\hat{\boldsymbol{x}}}=\boldsymbol{A} \hat{\boldsymbol{x}}+\boldsymbol{B}\left[\hat{\boldsymbol{F}}\left(\hat{\boldsymbol{x}} \mid \Theta_{1}\right)\right. \\
& \left.+\hat{\boldsymbol{G}}\left(\hat{\boldsymbol{x}} \mid \boldsymbol{\theta}_{2}\right) \varphi-\boldsymbol{u}_{\boldsymbol{a}}-\boldsymbol{u}_{\boldsymbol{b}}-\boldsymbol{u}_{\boldsymbol{s}}\right]+\boldsymbol{K}_{\boldsymbol{o}}\left(\boldsymbol{y}-\boldsymbol{C}^{T} \hat{\boldsymbol{x}}\right) \\
& \hat{\boldsymbol{y}}=\boldsymbol{C}^{T} \hat{\boldsymbol{x}}
\end{aligned}
$$

where $\mathbf{K}_{\mathbf{0}}$ is the observer gain matrix to guarantee the characteristic polynomial $\mathbf{A}-\mathbf{K}_{\mathbf{0}} \mathbf{C}$ to be Hurwitz, $\mathbf{u}_{\mathbf{a}}$ is a $H^{\infty}$ robust control to attenuate the disturbance effect on system outputs, $\mathbf{u}_{\mathbf{b}}$ is the feedback control for $\hat{\mathbf{e}}$ and $\mathbf{u}_{\mathbf{s}}$ is a sliding-mode control to compensate fuzzy approximation errors.

Define the observation error as $\widetilde{\mathbf{e}}=\mathbf{e}-\hat{\mathbf{e}}$ and $\widetilde{\mathbf{y}}=\mathbf{y}-\hat{\mathbf{y}}$ and subtracting (11) from (7) results in

$$
\begin{aligned}
& \dot{\tilde{\boldsymbol{e}}}=\left(\boldsymbol{A}-\boldsymbol{K}_{\boldsymbol{o}} \boldsymbol{C}^{T}\right) \tilde{\boldsymbol{e}}+\boldsymbol{B}\left[\left(\boldsymbol{F}(\boldsymbol{x})-\hat{\boldsymbol{F}}\left(\hat{\boldsymbol{x}} \mid \boldsymbol{\Theta}_{1}\right)\right)\right. \\
& \left.+\left(\boldsymbol{G}(\boldsymbol{x})-\hat{\boldsymbol{G}}\left(\hat{\boldsymbol{x}} \mid \boldsymbol{\theta}_{2}\right)\right) \varphi+\boldsymbol{u}_{a}+\boldsymbol{u}_{\boldsymbol{b}}+\boldsymbol{u}_{s}\right] \\
& \tilde{\boldsymbol{y}}=\boldsymbol{C}^{T} \tilde{\boldsymbol{e}}
\end{aligned}
$$

The adaptive fuzzy output feedback controller is designed as

$$
\begin{aligned}
& v=\hat{\boldsymbol{G}}\left(\hat{\boldsymbol{x}} \mid \boldsymbol{\theta}_{2}\right)^{-1} \\
& {\left[-\hat{\boldsymbol{F}}\left(\hat{\boldsymbol{x}} \mid \boldsymbol{\Theta}_{1}\right)+\boldsymbol{y}_{\boldsymbol{m}}{ }^{(3)}+\boldsymbol{K}_{c}{ }^{\boldsymbol{T}} \hat{\boldsymbol{e}}+\boldsymbol{u}_{\boldsymbol{a}}+\boldsymbol{u}_{\boldsymbol{b}}+\boldsymbol{u}_{\boldsymbol{s}}\right]}
\end{aligned}
$$

where $\mathbf{K}_{\mathbf{c}}{ }^{\mathbf{T}}$ is the feedback control gain vector to make the characteristic polynomial of to be Hurwitz.

There exist positive-definite solutions $\mathbf{P}_{\mathbf{1}}$ and $\mathbf{P}_{\mathbf{2}}$ in the following Lyapunov equation and Riccati equation for the given positive-definite matrices $\mathbf{Q}_{\mathbf{1}}$ and $\mathbf{Q}_{\mathbf{2}}$ [11]. Since $\mathbf{P}_{\mathbf{2}} \mathbf{B}=\mathbf{C}$ and $\widetilde{\mathbf{e}}$ is measurable, take the control law as 


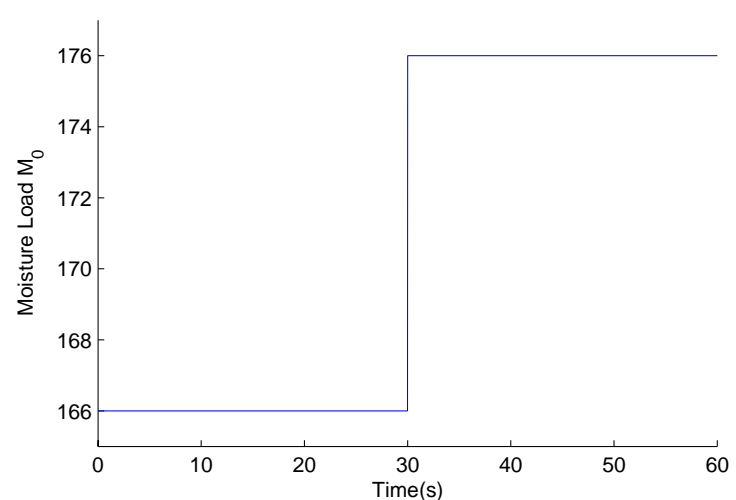

(a)

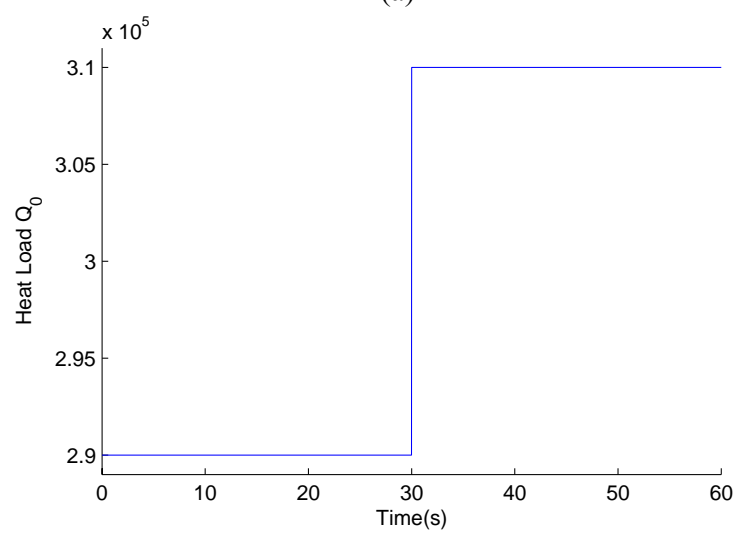

(b)

Fig. 2 Heat Load and Moisture Load.

(a) Heat Load, (b) Moisture Load

Table 1 Initial values for Simulation of HVAC system

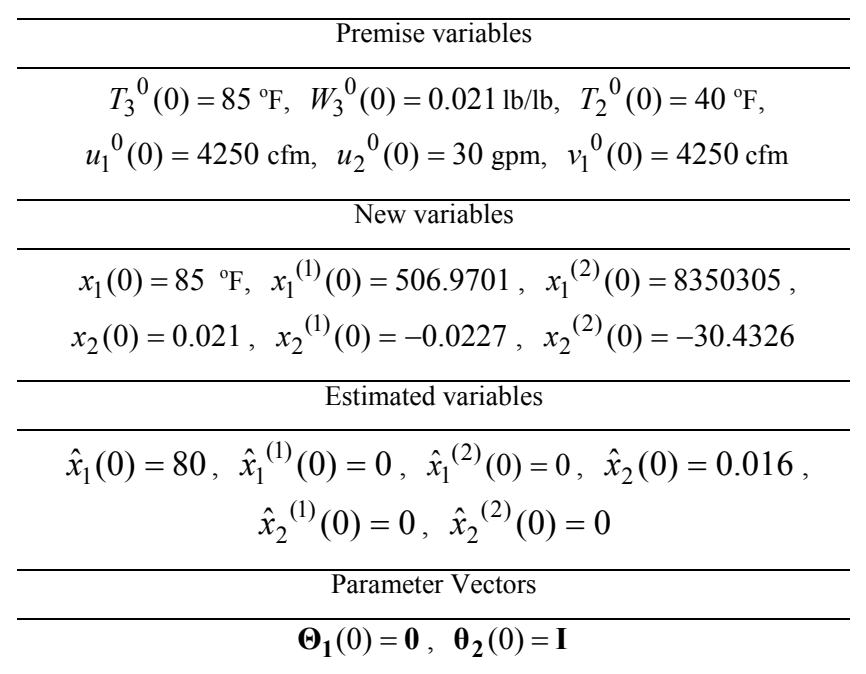

$$
\begin{aligned}
& \boldsymbol{v}_{\boldsymbol{a}}=-\frac{1}{2} \boldsymbol{R}^{-\boldsymbol{I}} \boldsymbol{B}^{\boldsymbol{T}} \boldsymbol{P}_{2} \tilde{\boldsymbol{e}}=-\frac{1}{2} \boldsymbol{R}^{-\boldsymbol{I}} \boldsymbol{C}^{T} \tilde{\boldsymbol{e}} \\
& \boldsymbol{v}_{\boldsymbol{b}}=-\boldsymbol{K}_{\boldsymbol{o}}{ }^{T} \boldsymbol{P}_{\boldsymbol{I}} \\
& \boldsymbol{v}_{\boldsymbol{s}}=-k \operatorname{sgn}\left(\boldsymbol{B}^{T} \boldsymbol{P}_{2} \tilde{\boldsymbol{e}}\right)=-k \operatorname{sgn}\left(\boldsymbol{C}^{T} \tilde{\boldsymbol{e}}\right)
\end{aligned}
$$

with $k>0$ as a sliding gain to be determined.
The parameter vector adaptive adjusting laws are chosen as

$$
\begin{aligned}
& \dot{\boldsymbol{\Theta}}_{1}=-\lambda_{1} \boldsymbol{\Phi}(\hat{\boldsymbol{x}})^{T}\left(\boldsymbol{B}^{T} \boldsymbol{P}_{2} \tilde{\boldsymbol{e}}\right)=-\lambda_{1} \boldsymbol{\Phi}(\hat{\boldsymbol{x}})^{T}\left(\boldsymbol{C}^{T} \tilde{\boldsymbol{e}}\right) \\
& \dot{\boldsymbol{\theta}}_{2}=-\lambda_{2} \boldsymbol{\Phi}(\hat{\boldsymbol{x}})^{T}\left(\boldsymbol{B}^{T} \boldsymbol{P}_{2} \tilde{\boldsymbol{e}} \varphi\right)=-\lambda_{2} \boldsymbol{\Phi}(\hat{\boldsymbol{x}})^{T}\left(\boldsymbol{C}^{T} \tilde{\boldsymbol{e}} \varphi\right)
\end{aligned}
$$

where $\lambda_{1}>0$ and $\lambda_{2}>0$ are two adaptation gains to be designed.

\section{Simulations}

In this simulation, we apply the adaptive fuzzy output feedback controller (13) to the continuous HVAC dynamics (7). In Table 1, the initial premise variables, the initial new variables, the initial estimated values and parameter vectors are considered. Fig. 2 shows the change in head load and moisture load.

$z_{1}$ and $z_{2}$ are used as the premise variables for the fuzzy system. Temperature is classified into four spheres between $4{ }^{\circ} \mathrm{F}$ and $149^{\circ} \mathrm{F}$. Humidity ratio also is classified into four spheres between $0.015 \mathrm{lb} / \mathrm{lb}$ and $1 \mathrm{lb} / \mathrm{lb}$. We choose the sixteen fuzzy rules which are made by each divided temperature and humidity ratio sphere pairs. The membership functions are chosen as

$$
\begin{aligned}
& \mu_{A_{1}^{1}}\left(z_{1}\right)=\left\{\begin{array}{cc}
1 & z_{1}<-30 \\
1-2\left(1+\frac{z_{1}}{30}\right)^{2} & -30<z_{1}<-1 \\
2\left(\frac{z_{1}}{30}\right)^{2} & -15<z_{1}<0 \\
0 & z_{1}>0
\end{array}\right. \\
& \mu_{A_{1}^{2}}\left(z_{1}\right)=\frac{1}{e^{z_{1}^{2} / 2^{*} 10^{2}}, \mu_{A_{1}^{3}}\left(z_{1}\right)=\frac{1}{\left(z_{1}-30\right)^{2} / 2^{*} 0^{2}}} \\
& \mu_{A_{1}^{4}}\left(z_{1}\right)=\frac{z_{2}<-0.015}{1+e^{-35\left(z_{1}-65\right)}} \\
& \mu_{A_{2}^{1}}\left(z_{2}\right)=\left\{\begin{array}{cc}
1 \\
1-2\left(1+\frac{z_{2}}{0.015}\right)^{2} \quad-0.015<z_{2}<-0.0075
\end{array}\right. \\
& \mu_{A_{2}^{2}}\left(z_{2}\right)=\frac{-0.0075<z_{2}<0}{\left.e^{z_{2}^{2} / 2 * 0.001^{2}}, \mu_{A_{2}^{3}}\left(z_{2}\right)=\frac{z_{2}}{0.015}\right)^{2}} e^{\left(z_{1}-0.01\right)^{2} / 2^{*} 0.001^{2}} \\
& \mu_{A_{2}^{4}}\left(z_{2}\right)=\frac{1}{1+e^{-0.0075\left(z_{2}-0.00175\right)}} \quad \begin{array}{c}
1 \\
0
\end{array}
\end{aligned}
$$

On constructing fuzzy systems $\hat{\mathbf{F}}\left(\hat{\mathbf{x}} \mid \boldsymbol{\Theta}_{\mathbf{1}}\right)$ and $\hat{\mathbf{G}}\left(\hat{\mathbf{x}} \mid \boldsymbol{\theta}_{\mathbf{2}}\right)$, and by using observer (11), we obtain the estimated $\hat{\mathbf{x}}$ and fuzzy systems $\hat{\mathbf{F}}\left(\hat{\mathbf{x}} \mid \boldsymbol{\Theta}_{\mathbf{1}}\right)$ and $\hat{\mathbf{G}}\left(\hat{\mathbf{x}} \mid \boldsymbol{\theta}_{\mathbf{2}}\right)$. Adaptation adjusting factors are chosen as $\lambda_{1}=0.0001$ and $\lambda_{2}=0.00001$ For the given $\mathbf{Q}_{\mathbf{1}}=\mathbf{Q}_{\mathbf{2}}=\operatorname{diag}\left[\mathbf{I}_{3 \times 3}, \mathbf{I}_{3 \times 3}\right]$, two positive-definite 


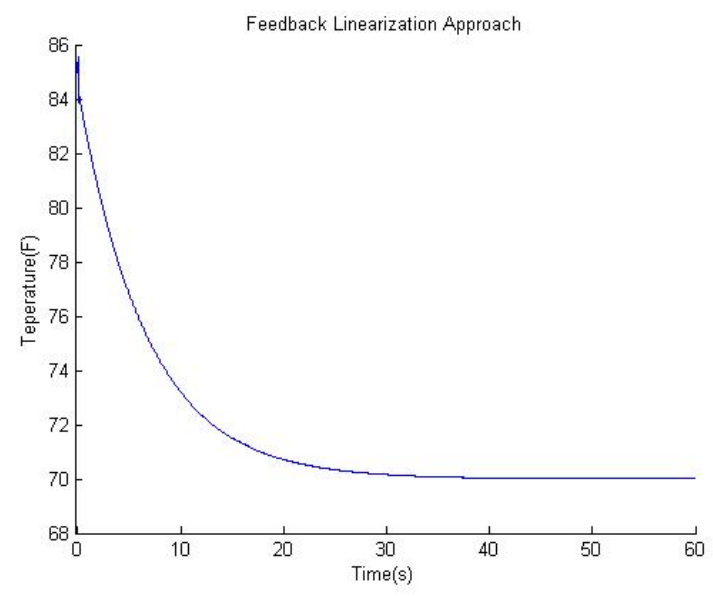

(a)

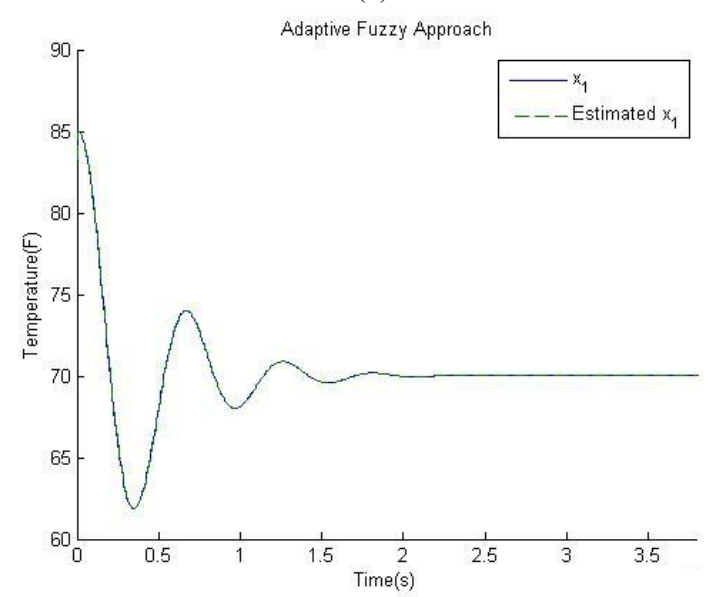

(b)



(c)

Fig. 3. Temperature Response for HVAC system.

(a) Adaptive Feedback Approach, (b) Adaptive Fuzzy Approach, (c) Adaptive Fuzzy Output Feedback Control Input.

matrices are solved from following Lyapunov equation and Riccati equation in [11]. The feedback and observer gain matrices are chosen as

$$
\mathbf{K}_{\mathbf{c}}=\operatorname{diag}\left(\left[\begin{array}{lll}
100 & 1000 & 10
\end{array}\right],\left[\begin{array}{lll}
10 & 1000 & 100
\end{array}\right]\right),
$$

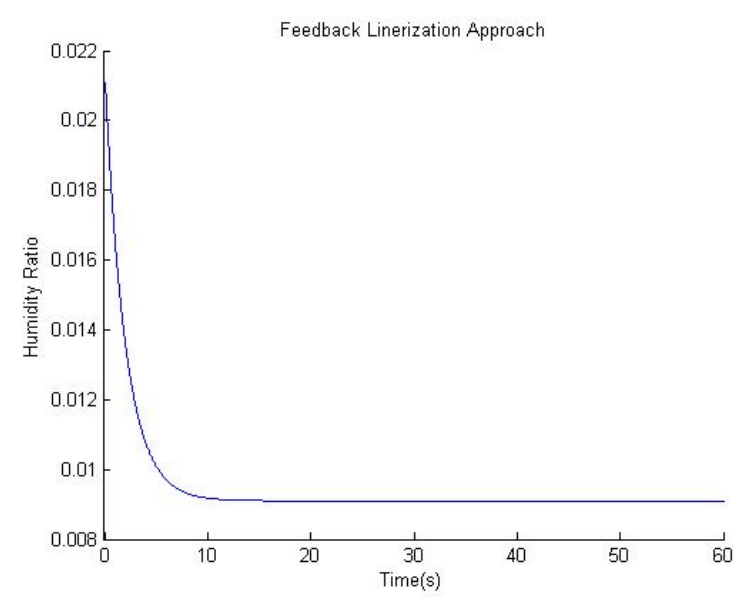

(a)

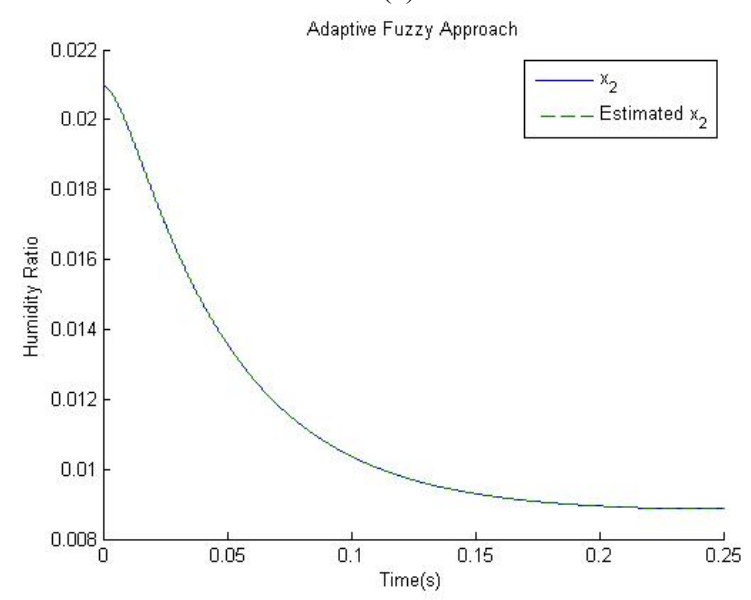

(b)

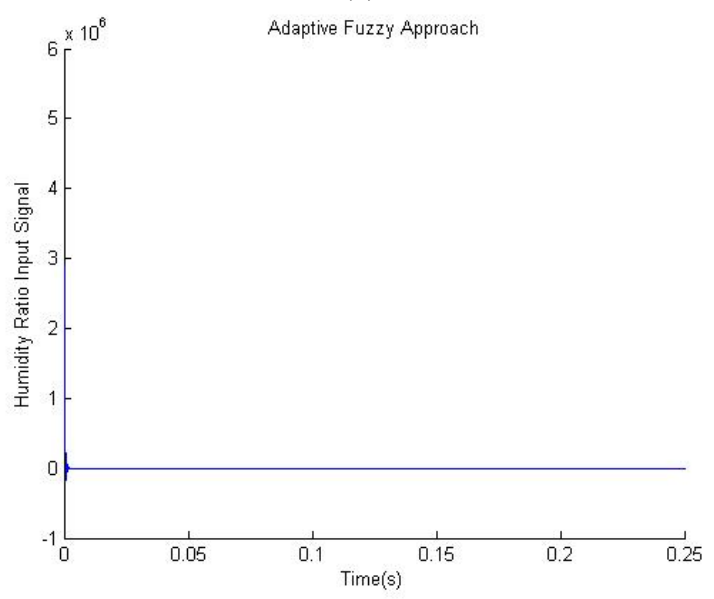

(c)

Fig. 4. Humidity Ratio Response for HVAC system. (a) Adaptive Feedback Approach, (b) Adaptive Fuzzy Approach, (c) Adaptive Fuzzy Output Feedback Control Input.

$$
\mathbf{K}_{\mathbf{o}}^{\mathbf{T}}=\operatorname{diag}\left(\left[\begin{array}{lll}
500000 & 15 & 7
\end{array}\right],\left[\begin{array}{llll}
450000 & 10 & 9
\end{array}\right]\right) .
$$

We want to track the temperature and humidity ratio to their respecting the given references of $70{ }^{\circ} \mathrm{F}$ and $0.0088 \mathrm{lb} / \mathrm{lb}$. Fig. 3 shows temperature response. Humidity Ratio response is shown 
as Fig. 4. Specially, Fig. 3(a) and Fig. 4(a) shows the results of the adaptive feedback linearization approach for HVAC system [9]. Fig. 3(b), Fig. 3(c), Fig. 4(b) and Fig. 4(c) is the results of the proposed adaptive fuzzy output feedback approach. The response of adaptive fuzzy output feedback control based on observer is much better and faster than adaptive feedback approach.

\section{Conclusions}

In this paper, we apply the feedback linearization technique with dynamic extension and design an adaptive fuzzy output feedback control based on observer for a nonlinear HVAC system with unavailable state variables. Since the state variables of nonlinear HVAC system is assumed to be unavailable, the state observer is designed to estimate state variables, via which fuzzy control schemes are formulated. Besides, we construct membership functions for the premise state variables to make the fuzzy logic system. The adaptive fuzzy output feedback controller tracks the desired temperature and humidity ratio to keep the comfort of thermal space. The adaptive fuzzy system behaves a HVAC system to maintain a desired track-points. Simulations have proved that the adaptive fuzzy output feedback controller is superior to the adaptive feedback control due to the ability of adaption to the estimated state variables. It is also possible for the adaptive fuzzy output feedback controller to retrench overconsumption of energy.

\section{References}

[1] Betzaida Argello-Serrano and Miguel Vlez-Reyes, "Nonlinear Control of a Heating, Ventilating and Air Conditioning System with Thermal Load Estimation," IEEE Trans. Control Systems Technology, Vol. 7, pp. 56-63, 1999.

[2] Rahmat Shoureshi, "Intelligent control systems : Are they for real?," Trans. ASME, Vol. 115, pp. 392-401, 1993.

[3] MohammadYaqub and SyedM.Zubair, "Capacity Control for Refrigeration and Air-Conditioning Systems: A Comparative Study", Journal of Energy Resources Technology, Vol. 123, pp. 92-9, 2001.

[4] Behzad Moshiri and Farzan Rashidi, "Self-tuning Based Fuzzy PID Controllers : Application to Control of Nonlinear HVAC Systems," IDEAL, Vol. 3177, pp. 437-442, 2004.

[5] Michael J. Coffin, Direct Digital Control for Building HVAC Systems. 2nd ed. [S.l.], Kluwer Academic Publishers, 1998.

[6] Elham Semsar, Mohammad Javad Yazdanpanah and Caro Lucas, "Nonlinear Control and Disturbance Decoupling of an HVAC system via Feedback Linearization and BackStepping," Proceedings of IEEE Conference, Vol. 1, pp. 646650, 2003.

[7] Farzan Rashidi and Behzad Moshiri, "Design of a Robust
Sliding Mode Fuzzy Controller for Nonlinear HVAC Systems," ICAISC 2004, LNAI 3070, pp. 984-989, 2004.

[8] N. Sheikholeslami, D. Shahmirzadi, E. Semsar, C. Lucas and M.J. Yazdanpanah, "Applying brain emotional learning algorithm for multivarizble control of HVAC systems," Journal of Intelligent and Fuzzy Systems, Vol. 17, pp. 35-46, 2006.

[9] Ming-Li Chiang and Li-Chen Fu, "Adaptive and Robust Control for Nonlinear HVAC System," the IEEE International Conference on Systems, Man, and Cybernetics, Vol. 6, pp. 4982-4987, 2006.

[10] C.P. Underwood, HVAC Control Systems : Modeling, analysis and design, E\&FN SPON, 1999.

[11]Han-Xiong Li and Shaocheng Tong, "A Hybird Adaptive Fuzzy Control for A Class of Nonlinear MIMO Systems," IEEE TRANS. ON FUZZY SYST., Vol. 11, pp. 24-34, 2003.

[12] Alberto Isidori, Nonlinear control systems, 3rd ed, New York - Springer Verlag, 1995.

Jaeho Baek He received his B.S degree in the Department of Control and Instrumentation Engineering from Daegu University, Korea in 2004. He received his M.S degree at the Department of Electrical and Electronics Engineering in Yonsei University, Korea in 2006. He is currently a Ph.D candidate in the Department of Electrical and Electronics Engineering from Yonsei University, Korea. His research interests include nonlinear systems, chaotic systems, neural networks control, fuzzy control, robot control, etc.

E-mail : jhbaek97@yeics.yonsei.ac.kr

Eun-ju Hwang She received her B.S degree in the Department of Metallurgical Engineering and Material Science from Hongik University, Korea in 1999. She received her M.S degree at the Department of Electrical Engineering in Yonsei University, Korea in 2006. She is currently a Ph.D candidate in the Department of Electrical and Electronics Engineering from Yonsei University, Korea.

E-mail : garung@yeics.yonsei.ac.kr

Euntai Kim He received the B.S. (summa cumlaude) and the M.S. and the Ph.D. degrees in electronic engineering, all from Yonsei University, Seoul, Korea, in 1992, 1994 and 1999, respectively. From 1999 to 2002, he was a full-time lecturer in the Department of Control and Instrumentation Engineering at Hankyong National University, Kyonggi-do, Korea. Since 2002, he has joined the faculties of the Department of Electrical and Electronic Engineering at Yonsei University, where he is currently an assistant professor. 
E-mail : etkim@yonsei.ac.kr

Mignon Park He received the B.S. degree and M.S. degree in electronics from Yonsei University, Seoul, Korea, in 1973 and 1977, respectively. He received the Ph.D. degree in University of Tokyo, Japan, 1982. He was a researcher with the Institute of Biomedical Engineering, University of Tokyo, Japan, from 1972 to 1982, as well as at the Massachusetts Institute of Technology, Cambridge, and the University of California Berkeley, in 1982. He was a visiting researcher in Robotics Division, Mechanical Engineering Laboratory, Ministry of International Trade and Industry, Tsukuba, Japan, from 1986 to 1987. He has been a Professor in the Department of Electrical and Electronic Engineering in Yonsei University, since 1982. His research interests include fuzzy control and application, robotics, and fuzzy biomedical system.

E-mail : mignpark@yonsei.ac.kr 